\title{
L'e-story ou le nouveau mythe hollywoodien
}

Claudia Moatti

Qu'est-ce que la mondialisation et les nouvelles technologies font à l'écriture de l'histoire ? Cette question légitime, que David Armitage et Jo Guldi soumettent à notre réflexion, bien des historiens se la posent depuis une dizaine d'années. Elle ne constitue ici, toutefois, qu'une captatio benevolentiae, car il s'agit avant tout pour les auteurs de lancer un «manifeste ». Rejetant sans distinction tous les turns, désormais dépassés, ils en appellent à un retour à une «nouvelle longue durée», que justifie, selon eux, l'abondance de sources mises à la disposition des chercheurs par la digitalisation ; retour nécessaire également pour remédier à la «crise morale » qui toucherait la discipline historique depuis les années 1970. Pourtant, ils ne nous proposent ni de réfléchir aux conséquences des transformations sociétales que traverse le monde contemporain, ni même d'analyser la nature de cette «crise ». Décrite dans ses manifestations extérieures (une extrême spécialisation de la recherche centrée sur la «courte durée », un éloignement des historiens de la sphère publique, un enfermement de la discipline sur elle-même), celle-ci est objectivée sans que soient prises en compte les attentes de l'époque ni celles d'aujourd'hui en termes de savoir et de quête de sens. Malgré leur ton péremptoire et oraculaire, une stratégie de la répétition et un sens aigu de l'autopromotion, D. Armitage et J. Guldi ne convainquent pas. Ils nous semblent plutôt poser de fausses questions, et leur propos révèle une régression philosophique et une disparition de la pensée critique.

\section{Longue durée ou temps long}


D. Armitage et J. Guldi plaident pour la «longue durée », dont on attend en vain une définition. Ils annoncent néanmoins qu'il ne s'agit pas d'un retour pur et simple au concept élaboré par Fernand Braudel. À la lecture de l'impressionnante bibliographie, on en déduit que leur manifeste n'a pas grand-chose à voir avec l'histoire lente et structurale, mais qu'il concerne plus banalement le temps long et « des questions d'envergure ».

Les auteurs appuient leur démonstration sur une longue revue de la production historiographique depuis l'Antiquité. La collection des titres, orientée vers le présent, forme un grand récit qui remplace la réflexion sur les modes de pensée et vise à persuader sans démontrer. Hérodote et Thucydide (qui, contrairement à ce qui est dit, écrivit une histoire événementielle, La guerre du Péloponnèse), F. Braudel et Thomas Piketty sont convoqués comme autant d'arguments d'autorité. L'histoire du temps long ne se justifie au fond que de ce qu'elle a existé, au mépris de toutes les distinctions, c'est-à-dire sans que l'on questionne le moins du monde la différence entre histoire universelle, deep, big, global ou world history, sans que l'on tienne non plus aucun compte des débats théoriques et des enjeux épistémologiques et institutionnels qui ont entouré l'œuvre de F. Braudel. La réflexion sur la langue, les catégories sociales, les concepts (Michel Foucault, Jacques Derrida, Roland Barthes, puis le Linguistic Turn), ou sur la notion de réalité historique (Henri-Irénée Marrou, Paul Veyne), tout est jeté aux oubliettes.

Cela s'explique fort bien: non pas par une quelconque ignorance mais par une idéologie aisément repérable, selon laquelle la technologie remplace la pensée en ce qu'elle détermine la méthode (la collection des données), l'écriture (le temps long) et le projet (plaire au public), en ce qu'elle permet de trouver des réponses à tous les problèmes posés auparavant. Gérard Noiriel analysait jadis la «crise de l'histoire » 
comme le résultat de l'anxiété théorique des historiens tentés par la philosophie ${ }^{1}$. L'article nous rassure sur ce point : l'épistémologie est loin et, avec elle, la transmission de tout un savoir accumulé. Sous une nouvelle forme, le positivisme et le scientisme sont de retour. Dévoilant leur vision bourgeoise de l'histoire, celle des grandes idées essentialisées, les auteurs saluent même ceux qui, avant eux, ont mis au jour les «vérités éternelles sur la dynamique des choses ${ }^{2} \gg$.

\section{Le quantitative turn et le temps long}

Par un mouvement pseudo-hégélien, ce retour au temps long est présenté comme un progrès. Tel en est l'idéologème : si, jadis, ce choix chronologique contraignait les historiens à recourir à des ouvrages de seconde main et si, par réaction, l'histoire courte fut centrée sur un corpus restreint d'archives, désormais, grâce à la «quantité astronomiques d'archives » et à l'aide des nouveaux logiciels («qui ne posent plus de problèmes »), la synthèse entre les deux est possible ${ }^{3}$.

Sans doute la digitalisation peut-elle susciter des questions nouvelles et incite-t-elle le chercheur à dépasser le cadre local déterminé jadis par la nature même des fonds d'archives, mais en quoi cela conduirait-il nécessairement au choix du temps long ? Outre les problèmes que posent la constitution, la contextualisation et la provenance de ces archives digitalisées, qui ne sont pas du tout abordés par D. Armitage et J. Guldi, doit-on rappeler que les archives ne parlent pas d'elles-mêmes ? Que les sources ne sont pas des documents neutres et bruts qu'il suffirait de réunir pour décrire ou interpréter la

1 - Gérard NoIRIEL, Sur la « crise » de l'histoire, Paris, Belin, 1996.

2 - David ARMitAge et Jo Guldi, «Le retour de la longue durée : une perspective angloaméricaine », Annales HSS, 70-2, 2015, p. .

3 - Ibid., p. . 
« réalité » passée ? Il est vrai qu'un grand nombre de statistiques sont mises à la disposition des chercheurs et que certains logiciels facilitent le travail d'inventaire, mais ce sont de simples outils. Les systèmes de gestion de bases de données (SGBD) qui, depuis une trentaine d'années, ont envahi la production scientifique archéologique peuvent bien accélérer l'étude comparative de monnaies ou de céramiques, ils ne peuvent rien d'autre, ni pour construire l'objet de recherche, ni pour réaliser des typologies, encore moins pour en tirer une analyse historique ${ }^{4}$. Si la digitalisation des sources et les moteurs de recherche procurent à l'évidence un gain de temps, ils ne sont utiles qu'aux spécialistes et encouragent plutôt la collaboration de chercheurs sur un domaine précis que la réalisation de grandes synthèses par un auteur unique.

L'éloge de la technique, devenu idéologie, marque en fait un retour aux objets et aux choses contre les idées et les discours qui font sens, aux explications générales au lieu d'une attention aux plis et à l'épaisseur de l'histoire ; au « réel »- les auteurs veulent comprendre «l'écosystème intellectuel » - au détriment de la conscience qu'en ont les différents acteurs de 1'histoire ${ }^{5}$. Chez Eric Hobsbawm, remarquait Enzo Traverso, il y a «d'une part l'historien social [celui de Bandits] qui s'intéresse à ceux 'd'en bas' en restituant leur voix et, de l'autre, l'auteur des grandes synthèses où les classes subalternes redeviennent une masse anonyme ${ }^{6} \gg$.

Nos auteurs seraient-ils atteints du syndrome hollywoodien - effets spéciaux et grandes productions - en s'en prenant aux formes d'analyse jugées trop artisanales et

4 - Julien ChANTEAU, «L'archéologie virtuelle », Médium, 35-2, 2013, p. 95-111.

5 - Quentin SkINNER, The Return of Grand Theory in the Human Sciences, Cambridge, Cambridge University Press, 1985, p. 184.

6 - Enzo Traverso, «Le siècle de Hobsbawm », Revue internationale des livres et des idées, 10, 2009, 11-16. 
besogneuses? Rien ne serait assez grand, assez long pour l'historien des temps nouveaux, cet esprit capable enfin grâce à sa maîtrise technique d'embrasser tout l'univers. À bas la critical theory ou la microstoria. Il faut désormais du colossal, de la big thought.

Il est souvent salutaire d'élargir son champ de recherche, et certains sujets (le climat, l'alimentation) se prêtent assurément au temps long, mais s'opposer à la synchronie au nom de la diachronie parait pour le moins simplificateur. Il faudrait plutôt s'interroger sur la durée et l'espace qui font sens pour telle ou telle enquête, définir «le champ spatial et temporel de l'intelligible » en histoire ${ }^{7}$, se demander ce qui fait l'unité d'une question, ce qui constitue un ensemble. Pour étudier les fondements culturels de l'Occident, l'historien peut remonter jusqu'à la Grèce, à Rome ou à la culture biblique, mais pas lorsqu'il cherche à comprendre la formation de l'idée européenne qui s'est constituée en tournant le dos à la Méditerranée et donc au monde classique. Le temps long n'est qu'une des voies de la recherche, certainement pas une norme.

À moins que ce projet ne manifeste rien d'autre que le symptôme d'une pensée hégémoniste, qui cherche à supprimer les aspérités et à globaliser le «temps », le naturalisant comme tous les objets qu'il englobe. Qu'on le veuille ou non, en effet, de même que l'écran d'ordinateur donne une image lisse de ce qui s'y présente malgré la multitude des pixels ${ }^{8}$, de même la technologie et le quantitatif qui lui est associé introduisent de la continuité dans ce qui est discontinu, de l'unité et de la généralité au

7 - Arnold J. ToYnBeE, The Study of History, vol. 1, Introduction: The Genesees of Civilizations, Oxford, Oxford University Press, 1934.

8 - Je renvoie à la réflexion de Jean-Yves CHEVALIER, «Le paradoxe du continu », Médium, 35-2, 2013, p. 52-71. 
détriment de la multipolarité et de la singularité. On proposera par exemple d'étudier l'histoire de la République ou de la Méditerranée, ou encore des classes sociales de l'Antiquité à nos jours, sans s'attarder sur la pertinence de ces catégories à travers le temps. Ainsi surgissent des universaux par lesquels on laisse croire que des institutions différentes remplissent «une fonction nécessaire à toute société humaine, et que dans cette permanence doit être cachée quelque mystérieuse finalité d'intégration de tout le corps social ${ }^{9} \gg$.

\section{L'engagement de l'historien}

On ne comprendrait pas toutefois en quoi le choix de la courte durée aurait eu pour conséquence mécanique la crise «morale » de l'histoire si l'on en restait à l'argument scientifique. L'objectif de ce manifeste est ailleurs et il est explicite : le «retour de la longue durée » devrait enfin permettre aux historiens de reprendre la place qu'occupent économistes ou futurologues sur la scène publique. L'historien de la «nouvelle longue durée », doté d'ambitions vertueuses, est tenu de produire des travaux utiles pour mieux enseigner, répondre à la demande des institutions ou réformer la société. Dans sa machine à remonter le temps, l'historien est en fait tourné vers l'avenir - comme si les objets étaient connus par avance.

Il est logique que ceux qui réfléchissent au pillage des ressources locales en Afrique aujourd'hui s'intéressent à l'histoire des empires modernes; comme il importe pour comprendre l'état présent de la démocratie en Europe de remonter le temps jusqu'au

9 - Paul VeYne, Comment on écrit l'histoire, Paris, Éd. du Seuil, 1978, p. 91. 
$\mathrm{XIX}^{\mathrm{e}}$ siècle ${ }^{10}$. Mais en quoi l'histoire de toutes les réformes agraires depuis les Gracques permettrait-elle d'inspirer une politique sociale dans le Chili d'aujourd'hui ?

Révélatrice est l'absence de distinction entre la recherche, l'enseignement et l'expertise publique ou privée. Il est vrai qu'aux États-Unis l'enseignement est davantage en phase avec les recherches dernière mode qu'en Europe, car il faut attirer le plus d'undergraduates possible - autant de jeunes clients pour les universités -, plaire aux mécènes et, dans la concurrence farouche entre les établissements, montrer qu'on est à l'avant-garde de l'innovation.

Il serait toutefois dommageable d'assimiler vulgarisation et recherche, ou de laisser entendre que l'histoire n'a pour fonction que de se placer sur le marché des idées, d'occuper une place institutionnelle, et non de répondre à une quête de sens. Dirait-on que l'art consiste à produire des œuvres qui puissent se vendre, à répondre à la demande des directeurs de musée ou à séduire le grand public ? Nierait-on qu'il consiste à trouver, à interroger la forme, comme le disait Witold Gombrowicz ?

Il faut indéniablement encourager au comparatisme, qui permet d'affiner le questionnement et, surtout (mais nos auteurs s'en soucient-ils ?), de mettre à l'épreuve dans le temps, collectivement, les catégories, les concepts, les questionnements, d'appréhender ainsi la particularité des modes de pensée ${ }^{11}$. Cela n'a rien à voir avec les vastes synthèses et les compilations écrites pour les décideurs ou le grand public, tel l'ouvrage de l'anthropologue David Graeber sur l'histoire de la dette depuis 5000 ans, une compilation intelligente et militante, passionnante dans ses premiers chapitres théoriques et réflexifs, ou dans ses analyses politiques du monde contemporain, mais

10 - Michèle Riot-SARCEy, La liberté en éclats, Paris, La Découverte, à paraître.

11 - Marcel Detienne, Comparer l'incomparable, Paris, Éd. du Seuil, 2000. 
d'une grande simplification sur les périodes les plus anciennes, chaque «âge » étant réduit à quelques idées générales naturalisées - qui ne font que conforter les postulats posés dans l'introduction ${ }^{12}$.

Poser la question de l'engagement en termes d'«utilité » et de «réponse institutionnelle » invite à une certaine circonspection quand elle émane d'un pays où l'histoire, l'anthropologie, la géographie n'ont pas toujours été au service d'un idéal de justice, mais ont souvent servi les institutions gouvernementales (les aires culturelles en sont un bon exemple), sinon parfois la CIA ${ }^{13}$. Elle nous interpelle d'autant plus aujourd'hui en Europe que les appels d'offres lancés par les institutions nationales ou européennes favorisent presque uniquement les laboratoires gigantesques et la recherche «appliquée ». Qu'adviendra-t-il à court terme des autres recherches, celles qui ne concernent aucun des grands sujets préalablement définis ? La curiositas fut dans l'Antiquité critiquée au nom d'un usage moral et politique du passé ${ }^{14}$. Sous des apparences de changement, la conception de l'histoire énoncée par D. Armitage et J. Guldi renvoie étrangement à ce modèle dépassé.

Écrire l'histoire ne consiste pas plus à collecter toutes les sources qu'à plaire aux institutions et au grand public, mais, comme l'écrivait Michel de Certeau, à transformer le matériau sélectionné pour en comprendre la spécificité, et en saisir l'historicité. C'est dire qu'elle engage un sujet et une pensée critique. On peut appeler e-storique la

12 - David Graeber, Dette : 5000 ans d'histoire, trad. par F. et P. Chemla, Paris, Éd. les liens qui libèrent, [2011] 2013.

13 - David H. PRICE, Weaponizing Anthropology: Social Science in Service of the Militarized State, Oakland, AK Press, 2011.

14 - CiCERON, De finibus, 5, 2, 5-6. 
réduction a priori de toute historicité et de toute critique dans la techno-chronologie et le marketing. Les e-storiens, on les voit venir de loin !

Claudia Moatti

Université de Paris 8 et University of Southern California 